\title{
CRIANÇAS E ADOLESCENTES COM DIABETES MELLITUS: VANTAGENS E LIMITES DA MONITORIZAÇÃO
}

[Monitorization to diabetic children and diabetic adolescents: advantages and limits]

Maria Helena Dantas de Menezes Guariente*
Márcia Eiko Karino**
Maria do Carmo Lourenço Haddad ${ }^{\star \star}$
Francismery Kasai**
Carolliny Rossi de Faria
Iria Roberta Staut Freitas

RESUMO: A monitorização do controle glicêmico é parte fundamental do tratamento do Diabetes Mellitus, especialmente em diabéticos tipo 1 , uma vez que o controle metabólico diminui ou até mesmo retarda complicações crônicas. Com o objetivo de avaliar a prática da monitorização glicêmica entre crianças e adolescentes diabéticos foi realizada uma pesquisa descritiva na abordagem quantitativa com diabéticos atendidos em um programa interdisciplinar do Ambulatório Hospital das Clínicas da Universidade Estadual de Londrina. Aplicou-se um instrumento sobre a freqüência de realização da monitorização e dificuldades encontradas para sua efetivação. A amostra constituiu-se de 63 diabéticos, sendo $47(74,6 \%)$ adolescentes e $16(25,4 \%)$ crianças. Concluiu-se que nessa população a monitorização está aquém do ideal. Esta informação mobiliza os profissionais da equipe multiprofissional na continuidade do trabalho em ressaltar a importância do controle glicêmico, auxiliando os diabéticos na busca de alternativas para a superação das dificuldades de ordem emocional, econômica e social, que dificultam a efetivação do procedimento.

PALAVRAS-CHAVE: Diabetes Mellitus; Criança diabética; Adolescente diabético; Glicemia; Monitorização ambulatorial.

\section{INTRODUÇÃO}

O Diabetes Mellitus é uma doença crônica assumindo atualmente proporções epidêmicas. Segundo a Federação Nacional de Associações de Diabéticos e Ministério da Saúde

* Docente do Departamento de Enfermagem da Universidade Estadual de Londrina e Coordenadora do Núcleo de Pesquisa em Enfermagem do Hospital Universitário Regional do Norte do Paraná.

** Docente do Departamento de Enfermagem da Universidade Estadual de Londrina e Universidade Norte do Paraná.

*** Acadêmicas do Curso de Graduação em Enfermagem / UEL

**** Enfermeira do Ambulatório do Hospital de Clínicas/UEL
(PASTORE, 1998), no Brasil há cerca de 9 milhões de diabéticos, dos quais apenas metade sabe que apresenta esta patologia, e destes, 23\% não se tratam. Em conseqüência disso, o índice de óbitos cresce assustadoramente, atingindo em 1995, 24 mil óbitos decorrentes do diabetes, o qual já corresponde a $4^{\circ}$ causa de morte no mundo (OMS, 1993), apud PASTORE (1998)

Diante dessas evidências, é importante ressaltar que apesar de se tratar de uma doença para qual a ciência ainda não encontrou a cura, complicações agudas e crônicas como o coma hipo ou hiperglicêmico, micro ou macroangiopatias bem como neuropatias são previnidas ou até mesmo evitadas através de um bom controle glicêmico.

Segundo os resultados do DCCT-Diabetes Control and Complication Trial Research Group (DUNNE et al., 1995), existe uma clara recomendação para que os diabéticos mantenham seus níveis de glicose no sangue tão próximo quanto possível dos níveis normais, para evitar complicações do diabetes a longo prazo.

Portanto, a monitorização do nível glicêmico é parte fundamental no tratamento do Diabetes Mellitus, especialmente em diabéticos do tipo 1 , os quais necessitam de doses diárias de insulina exógena ficando assim mais susceptíveis a possíveis descompensações glicêmicas. Diversos testes de controle podem ser realizados, tais como a glicosúria e a glicemia capilar, que fornecem valores imediatos a curto prazo e também a hemoglobina glicosilada que permite obter uma média dos níveis de glicose sangüínea durante período de tempo de 2 a 3 meses.

Entretanto, BRUNNER e SUDDARTH (2000) ressaltam que é importante que o método usado pelos pacientes esteja apropriado a seu nível de habilidade. Os fatores que interferem incluem a acuidade visual, coordenação motora fina, capacidade intelectual, conforto com a tecnologia, motivação e custo. 
Os profissionais da saúde, especialmente os enfermeiros, desempenham papel fundamental na assistência para a realização da monitorização, assim como na educação em saúde, permitindo que portadores de diabetes se tornem aptos a desenvolver tal procedimento em regime domiciliar de forma segura, adequada e acima de tudo de forma consciente. Segundo SOUZA e ZANETTI (2000), o enfermeiro é o profissional responsável pelo acompanhamento domiciliar das famílias com crianças e adolescentes diabéticos, comprometendo-se com a educação e o treinamento. Assim o diabético passa a avaliar os valores glicêmicos aferidos, suas implicações e providências a serem tomadas a fim de evitar a instalação de complicações crônicas, contribuindo para uma vida mais saudável e também para a redução dos custos com tratamento, hospitalizações e mortes precoces.

O Diabetes Mellitus é uma das principais causas clínicas de hospitalizações no Brasil, o que significa altos custos financeiros. Suas manifestações crônicas ou complicações são causas freqüentes de invalidez (BRASIL,1993). BRUNNER e SUDDARTH (2000) ressaltam ainda que as taxas de hospitalizações para pessoas com diabetes são 2,4 vezes maior para adulto e 5,3 vezes maior para as crianças do que para a população geral.

Atentos a este contexto e interessadas em implementar uma assistência à criança e ao adolescente diabético condizente às suas necessidades e prioridades nos indagamos: Como tem sido a prática da monitorização (glicosúria e glicemia capilar) entre estes sujeitos?

\section{OBJETIVOS}

\subsection{OBJETIVO GERAL}

- Avaliar a prática da monitorização glicêmica entre crianças e adolescentes diabéticos.

\subsection{OBJETIVOS ESPECÍFICOS}

- Determinar o tipo de teste realizado pelos pacientes diabéticos para o controle glicêmico;

- Relacionar a freqüência da realização dos controles glicêmicos a faixa etária dos entrevistados;

- Identificar o conhecimento dos portadores de diabetes em relação ao valor glicêmico normal;

- Verificar os motivos para a não realização da monitorização glicêmica
- Levantar com os portadores de diabetes ou familiares possíveis sugestões para a superação das dificuldades na realização da monitorização.

\section{METODOLOGIA}

Foi realizada uma pesquisa de caráter descritivo exploratória na abordagem quantitativa, no Ambulatório do Hospital das Clínicas (AHC), órgão suplementar da Universidade Estadual de Londrina.

O estudo focalizou diabéticos atendidos em um programa ambulatorial, desenvolvido por uma equipe de saúde multiprofissional.

A amostra foi constituída a partir dos seguintes critérios de inclusão: crianças e adolescentes na faixa etária de 0 a 20 anos com Diabetes Mellitus tipo 1, e que após explicação do objetivo da pesquisa e do seu livre direito em participar ou não, consentiram em responder o instrumento elaborado pelas pesquisadoras.

Foi aplicado um questionário com 10 questões abordando itens de identificação, conhecimentos sobre nível glicêmico normal, freqüencia do controle glicêmico domiciliar e levantamento de sugestões para superação das dificuldades na monitorização.

\section{RESULTADOS E DISCUSSÕES}

O Programa de Atendimento Ambulatorial ao paciente diabético do AHC atende em torno de 160 diabéticos. A população deste estudo foi composta por $81(50,6 \%)$ diabéticos, tendo como amostra 63 (77,8\%) indivíduos, os quais corresponderam aos critérios de seleção, sendo 16 $(25,4 \%)$ crianças e $47(74,6 \%)$ adolescentes, seguindo a preconização da Organização Mundial de saúde, que estabelece, para a faixa etária e ciclo vital, a idade de até 10 anos para crianças e de 10 a 20 anos de idade para adolescentes.

GRÁFICO 1-COMPOSIÇÃO DA AMOSTRA

37 $(58,7 \%)$

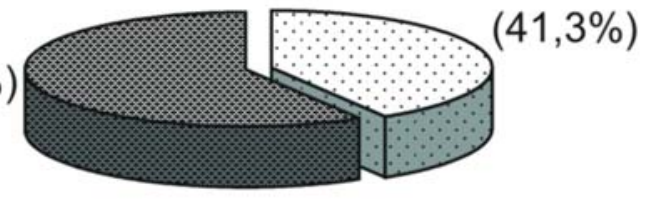

$\square$ masculino 圈feminino 
GRÁFICO 2-FREQÜÊNCIA DOS DIABÉTICOS, CONFORME SEXO

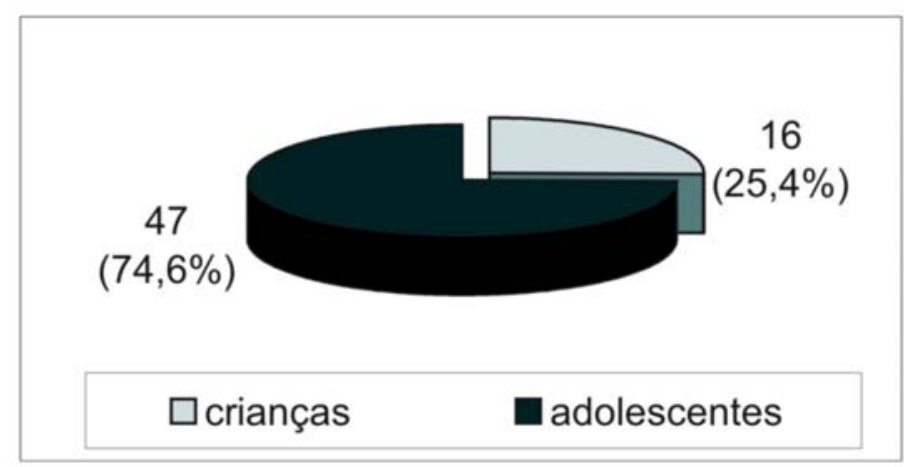

Dos diabéticos que participaram da pesquisa, 37 $(58,7 \%)$ são do sexo feminino e 26 (41,3\%) do sexo masculino.

TABELA 1 - DISTRIBUIÇÃO DAS CRIANÇAS E ADOLESCENTES, SEGUNDO TEMPO QUE POSSUEM DIABETES MELLITUS (EM ANOS).

\begin{tabular}{c|c|c}
\hline TEMPO (ANOS) & $f$ & $\%$ \\
\hline$<1$ & 3 & 4,8 \\
$1-3$ & 9 & 14,3 \\
$3-5$ & 21 & 33,3 \\
$5-7$ & 9 & 14,3 \\
$7-9$ & 6 & 9,5 \\
$9-11$ & 6 & 9,5 \\
$11-13$ & 2 & 3,3 \\
$13-15$ & 3 & 4,8 \\
$15-17$ & 1 & 1,6 \\
$17-19$ & 3 & 4,8 \\
\hline TOTAL & $\mathbf{6 3}$ & $\mathbf{1 0 0}$ \\
\hline
\end{tabular}

Quanto ao tempo de conhecimento do diagnóstico da doença, $21(33,3 \%)$ referiram saber há 3 a 5 anos, 9 (14,3\%) entre 1 a 3 anos, outros 9 (14,3\%) entre 5 a 7 anos. Observase ainda que $7(11,2 \%)$ adolescentes já convivem com esta patologia entre 13 a 19 anos.

O diabetes insulino-dependente atinge crianças e adolescentes na proporção de 1 para 500 e de 1 para 200 indivíduos, respectivamente, com a redução, em até 1/3 da expectativa de vida (BRASIL,1993).

Este perfil epidemiológico reforça a importância da detecção precoce da doença para um bom controle e qualidade de vida dos diabéticos.

\section{GRÁFICO 3 - PREVALÊNCIAAMOSTRAL QUANTO A MONITORIZAÇÃO} DOMICILIAR

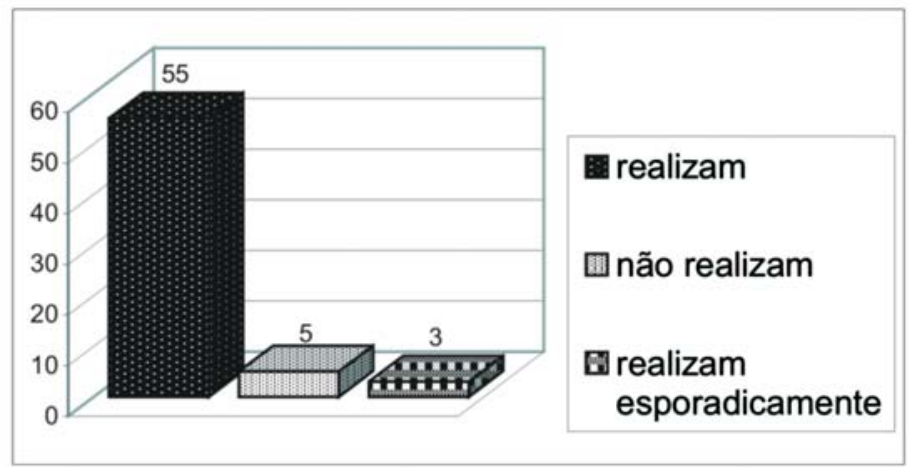

Quanto à realização da monitorização domiciliar, 55 $(87,3 \%)$ realizam, 5 (8\%) não realizam e $3(4,7 \%)$ realizam esporadicamente.

Dos 55 diabéticos que realizam a monitorização domiciliar, a maioria justificou esta conduta frente a necessidade de se obter um controle glicêmico frequente, outros realizam para saber a dosagem de insulina a ser aplicada ou ainda pela importância de sua realização devido a gravidez, ou por se tratar de uma doença que mal controlada leva a morte.

Os que relataram realizar esporadicamente, atribuíram esta atitude à falta de motivação e do esquecimento frente as atividades diárias.

A maioria dos que afirmaram não realizar nenhum tipo de teste de controle, justificaram o fato devido ao alto custo dos materiais. Outros relataram a falta de tempo e a "preguiça", apesar de ir ao médico regularmente para o acompanhamento do controle glicêmico. Havendo ainda um relato de não realizar por falta de colaboração da criança diabética.

A monitorização do controle glicêmico é essencial no tratamento do Diabetes Mellitus, tanto para reajustar esquemas terapêuticos quanto para prevenção de complicações agudas e crônicas desta patologia.

Segundo ZANETTI, MENDES e RIBEIRO (2001, p. 34), para atingir um bom controle metabólico do diabético na criança e no adolescente é "necessário melhorar o provimento de materiais e sobretudo envolver efetivamente a família neste tratamento". A atuação da família é um fator importante na redução de complicações para criança, propiciando condições de vida mais saudável. Para que a família participe do tratamento é necessário que aprenda a manusear os instrumentos e praticar o controle domiciliar diário do diabetes.

TABELA 2 - RELAÇÃO ENTRE A FAIXA ETÁRIA AMOSTRAL E A REALIZAÇÃO DO CONTROLE GLICEMMICO

\begin{tabular}{|c|c|c|c|c|c|c|c|}
\hline \multirow{2}{*}{$\begin{array}{l}\text { IDADE } \\
\text { (ANOS) }\end{array}$} & \multicolumn{2}{|c|}{ SIM } & \multicolumn{2}{|c|}{ NÃO } & \multicolumn{2}{|c|}{ AS VEZES } & \multirow{2}{*}{ TOTAL } \\
\hline & , & $\%$ & $f$ & $\%$ & $f$ & $\%$ & \\
\hline $2-4$ & 1 & 1,6 & 0 & 0 & 0 & 0 & 1,6 \\
\hline 4-6 & 3 & 4,7 & 0 & 0 & 0 & 0 & 4,7 \\
\hline $6-8$ & 4 & 6,3 & 1 & 1,6 & 0 & 0 & 7,9 \\
\hline 8-10 & 5 & 7,9 & 1 & 1,6 & 1 & 1,6 & 11,1 \\
\hline $10-12$ & 5 & 7,9 & 0 & 0 & 0 & 0 & 7,9 \\
\hline $12-14$ & 8 & 2,7 & 0 & 0 & 0 & 0 & 12,7 \\
\hline $14-16$ & 11 & 17,5 & 0 & 0 & 0 & 0 & 17,5 \\
\hline $16-18$ & 7 & 11,1 & 2 & 3,2 & 0 & 0 & 14,3 \\
\hline $18-20$ & 11 & 17,5 & 1 & 1,6 & 2 & 3,2 & 22,3 \\
\hline TOTAL & 55 & 87,3 & 5 & 8 & 3 & 4,8 & 100 \\
\hline
\end{tabular}

Ao analisarmos a freqüência da realização de testes para controle glicêmico, concomitantemente com a faixa etária, é possível verificar através da tabela 2 que a realização da monitorização tem predominância nas faixas etárias de 
14 à 16 anos e de 18 à 20 ambos com 11(17,5\%) diabéticos. ZANETTI et al. (2001), reforçam que mudar comportamentos frente às práticas de saúde é uma tarefa árdua e contínua, que exige dos profissionais de saúde, tempo, recursos e disponibilidade para instrumentalizar-se em termos de diabetes, de capacitação pedagógica, na busca de alternativas que poderão sensibilizar as crianças e adolescentes para o auto-cuidado e apoiar a família no cuidado domiciliar.

O tratamento é variável durante o curso da doença devido a fatores como mudança do estilo de vida, condições físicas e emocionais, bem como avanços nos métodos de tratamento oriundos da pesquisa. Segundo BRUNNER e SUDDARTH (2000, p. 878),

" o tratamento do diabetes envolve constante avaliação e modificações do plano de tratamento por profissionais de saúde, bem como ajustes diários na terapia do paciente e que embora a equipe de saúde conduza o tratamento, é o paciente que se defronta com a carga diária de prosseguir com os detalhes de um esquema terapêutico complexo. Por este motivo, a educação do paciente e seus familiares é vista como um componente essencial do tratamento do diabetes, com importância igual a outros componentes do esquema de tratamento".

GRÁFICO 4 - ÍNDICE DE ERROS EACERTOS COM RELAÇÃOAO VALOR GLICÊMICO CORRETO

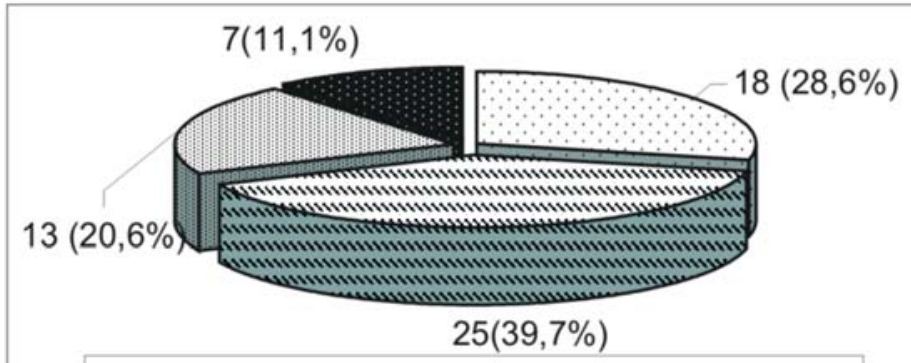

\section{Gacertos $\mathrm{⿴}$ erros $\square$ em parte $\mathbf{i}$ não sabe}

Ao serem questionados sobre o valor glicêmico normal, $25(39,7 \%)$ erraram tanto o valor mínimo quanto o máximo de glicose no sangue, $13(20,6 \%)$ acertaram em parte o valor glicêmico normal e apenas $18(28,6)$ acertaram completamente.

Constatamos no atendimento ambulatorial que o paciente diabético e mesmo a família não têm clareza de vários aspectos da doença como o valor normoglicêmico apesar deste ser um dos conteúdos explicados e reforçados pela equipe nas consultas do programa.

Ressaltamos contudo, ser indispensável que os diabéticos e familiares estejam, adequadamente orientados quanto aos valores glicêmicos normais e as implicações causadas pela descompensação glicêmica. Da mesma forma, saibam avaliar os resultados obtidos e através dos mesmos, a fim de tomar as devidas providências necessárias, sejam elas relacionadas a reajustes terapêuticos ou mudanças no estilo de vida.

Segundo DUNNE et al (1995, p.1) "a eficácia dos dados é essencial na decisão médica de mudar as doses de medicamento (insulina e sulfoniluréia) ou na determinação do sucesso ou fracasso de uma terapia por parte do profissional e saúde. Sem dados sobre a glicose no sangue médicos e pacientes não podem tomar decisões sobre as terapias prescritas". Assim, todos os profissionais de saúde devem atuar na educação de pacientes diabéticos a respeito da importância da automonitorização da glicose no sangue, como também no conhecimento dos valores normais de glicemia preconizados.

\section{GRÁFICO 5 - TIPO DE TESTE DE MONITORIZAÇÃO REALIZADO PELOS DIABÉTICOS}

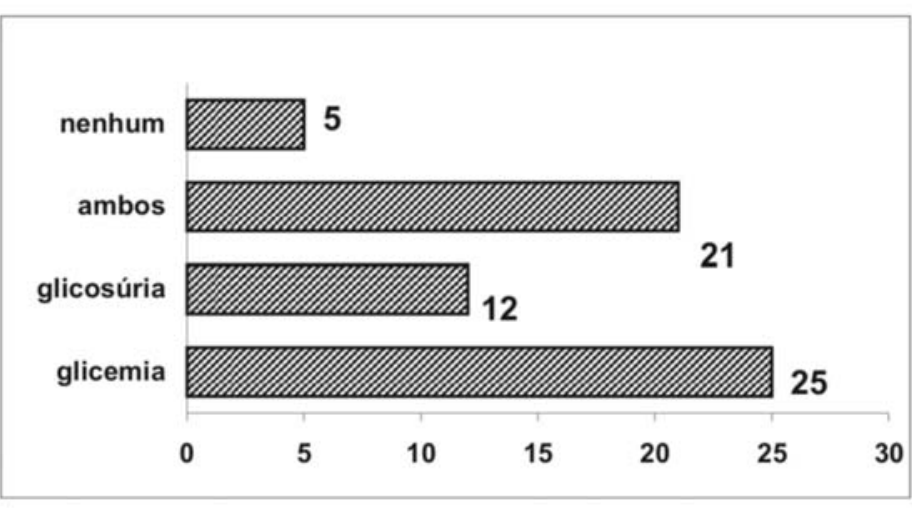

Quanto ao tipo de teste domiciliar para controle glicêmico realizado pelos diabéticos, verifica-se, através do gráfico 5 , que $25(39,7 \%)$ dos indivíduos realizam apenas glicêmia capilar, 12 (19\%) apenas a glicosúria, 21 (33,3\%) afirmaram realizar ambos os testes e 5 (8\%) nenhum dos testes.

O controle glicêmico através do teste de glicemia capilar, quando realizado corretamente, garante maior confiabilidade quanto aos níveis de glicemia no exato momento de sua aferição. Além disso, informam quanto a ocorrência tanto de um quadro de hiperglicemia ou hipoglicemia. No entanto, a manutenção deste tipo de teste representa um custo muito elevado, tornando-o na maioria das vezes inacessível aos diabéticos de baixa renda. Diante disso o diabético, passa a realizar o teste de glicosúria devido ao seu baixo custo. Entretanto, este teste apresenta muitas desvantagens, uma delas refere-se ao fato de detectar apenas os estados de hiperglicemia, não informando níveis hipoglicêmicos. Além de oferecer valores glicêmicos duvidosos quando não se respeita os critérios para coleta de 
urina para o teste. O que pode ser confirmado por GOLDTEIN et al. (1995), ao afirmarem que embora a monitorização da glicosúria constitua em um teste urinário útil no processo de autocontrole do diabetes, apresenta algumas limitações.

BRUNNER e SUDDARTH (2000, p. 884) confirmam que

Antes da disponibilidade dos métodos de automonitorização, o teste de glicose na urina era o único método disponível para monitorização diária do diabetes. Hoje seu uso está limitado aos pacientes que não podem ou não desejam fazer teste de glicose sangüíneo. Entretanto o teste de glicemia permite que as pessoas com diabetes sejam capazes de ajustar o esquema de tratamento para se obter um controle ótimo da glicose. Isto permite a detecção e prevenção de hipo e hiperglicêmia e tem papel crucial na normalização dos níveis de glicose sangüíneo o que possivelmente reduz as complicações diabéticas de longo prazo.

GRÁFICO 6 - FREQÜÊNCIA DE REALIZAÇÃO DA GLICEMIA CAPILAR

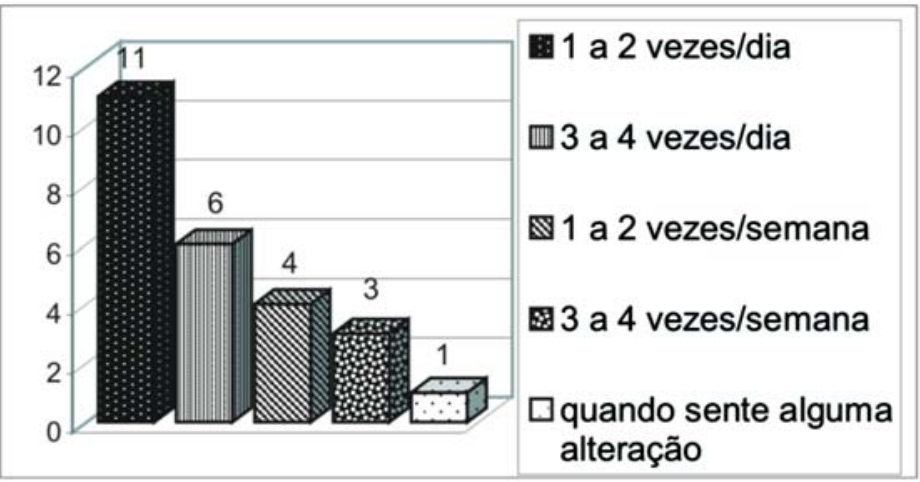

Em relação ao gráfico 6 podemos observar que 11 (44\%) realizam a glicemia capilar de 1 a 2 vezes/dia , 6 (24\%) de 3 a 4 vezes/dia e 4 (16\%) de 1 a 2 vezes/semana. Com base nestes dados, verifica-se que a freqüência de realização destes testes para alguns pacientes ainda não corresponde a recomendação da literatura, que preconiza o mínimo 4 vezes/dia.

Segundo DUNNE et al. (1995), as indicações e recomendações quanto a freqüência de monitorização dos níveis de glicose no sangue variam dependendo das situações clínicas. Para este autor, os pacientes que estão tentando atingir níveis de glicemia próximos do normal necessitam monitorar os níveis de glicose no sangue no mínimo 4 vezes ao dia. AAMERICAN DIABETES ASSOCIATION (1994) apud DUNNE et al. (1995), ressaltam que uma redução da freqüência da realização da monitorização para menos de 4 vezes ao dia resultará em uma piora do controle glicêmico.
Porém, observa-se que o alto custo dos materiais para a manutenção da monitorização aliado ao fato de se tratar de um teste invasivo, para muitos diabéticos, torna-se um obstáculo para sua adequada realização. Entretanto, cabe à equipe de saúde ressaltar constantemente a importância da aferição da glicemia várias vezes ao dia para a continuidade do tratamento, possibilitando o reajuste do esquema terapêutico quando necessário.

\section{GRÁFICO 7 - FREQÜÊNCIA DA REALIZAÇÃO DE GLICOSÚRIA}

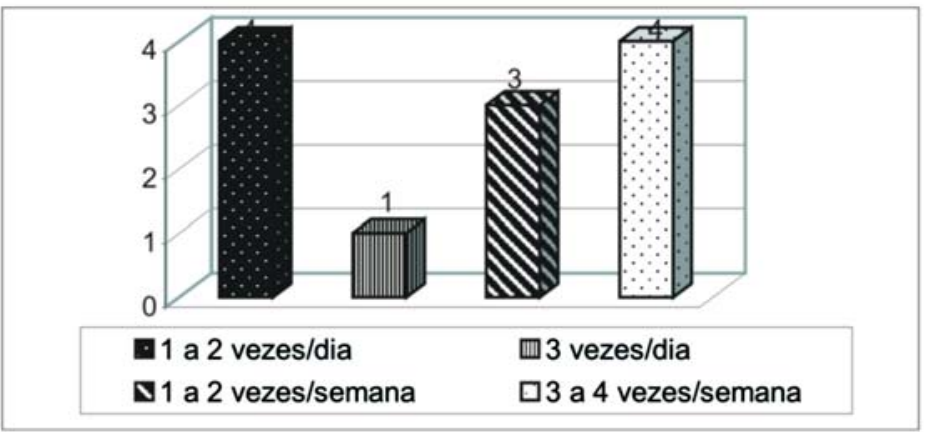

Através do gráfico 7, verifica-se que 4 (33,3\%) diabéticos realizam a glicosúria com freqüência de 1 a 2 vezes/dia, já outros $4(33,3 \%)$ a fazem somente de 3 a 4 vezes/semana. Diante disso, constata-se que ainda há uma grande deficiência quanto à adequada realização deste teste, pois ainda existe uma significativa parcela de diabéticos que a realizam em um número muito reduzido de vezes, já que este é um único teste de controle realizado pelos mesmos.

Segundo OLIVEIRA (1997, p. 38),

todos os diabéticos tipo I devem ser orientados para avaliar diariamente as glicosúrias três a quatro vezes ao dia (antes do café, antes do almoço, antes do jantar e ao deitar), principalmente quando estiverem descompensados ou em fase de adaptação de doses. Uma vez controlados podem fazer duas determinações diárias (uma determinação em jejum ou antes das refeições principais - outra determinação duas horas após uma refeição principal) duas ou três vezes por semana.

TABELA 3 - FREQÜÊNCIA DA REALIZAÇÃO DE AMBOS OS TESTES

\begin{tabular}{|c|c|c|}
\hline \multirow{2}{*}{ FREQÜÊNCIA DA REALIZAÇÃO DOS TESTES } & \multicolumn{2}{|c|}{ Nº DE DIABÉTICOS } \\
\hline & GLICÊMIA & GLICOSÚRIA \\
\hline 1 a 2 VEZES AO DIA & 2 & 12 \\
\hline 3 a 4 VEZES AO DIA & 3 & 4 \\
\hline 1 a 2 VEZES POR SEMANA & 4 & 3 \\
\hline 3 a 4 VEZES POR SEMANA & 10 & 2 \\
\hline MENSALMENTE & 2 & 0 \\
\hline TOTAL & 21 & 21 \\
\hline
\end{tabular}


Conforme a tabela 3, verifica-se que dos 21 diabéticos que realizam ambos os testes de controle glicêmico, em relação a glicosúria a maioria $12(57,14 \%)$ realiza o teste de 1 a 2 vezes ao dia, já os de glicemia a maioria $10(47,6 \%)$ realiza de 3 a 4 vezes por semana. Observa-se que apesar de realizarem ambos os testes, o teste com maior freqüência ao final de um mês é o teste de glicosúria já que é realizado pela maior parte dos pacientes diariamente, diferente da glicemia o qual poucas pessoas a realizam diariamente. Tal fato pode ser explicado pelo alto custo dos materiais para realização deste teste e o desconforto devido a punção necessária.

\section{GRÁFICO 8 - SUGESTÕES APRESENTADAS PELOS DIABÉTICOS PARA SUPERAR}

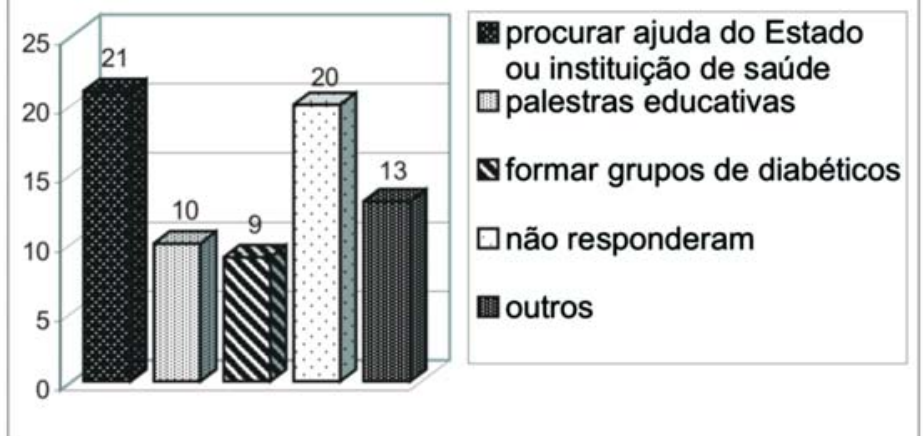

Procuramos ainda levantar com os portadores de diabetes e seus familiares possíveis sugestões para a superação das dificuldades da não realização da monitorização glicêmica. Através do gráfico 8, observa-se que 21 sugestões englobam a necessidade do governo, em especial a prefeitura, contribuir financeiramente com fornecimento de materiais ou redução dos preços dos mesmos; 10 sugeriram a realização de palestras educativas ou campanhas enfocando a importância monitorização, assim como as consequências decorrentes da sua não efetivação, através de depoimentos dos próprios diabéticos; 9 relataram que é indispensável a formação de grupos de diabéticos para aquisição de materiais com preços mais acessíveis, 13 referiram sugestões diversificadas como a necessidade de auto- conscientização a fim de que o próprio diabético aceite a doença e colabore com o tratamento, cortar as fitas dos testes o que possibilita a redução dos custos na compra dos materiais além de permitir a realização de maior número de testes, e 20 diabéticos não apresentaram nehuma alternativa para sanar as dificuldades quanto ao procedimento.

\section{CONCLUSÃO}

A automonitorização glicêmica é parte fundamental no tratamento do Diabetes Mellitus, uma vez que tal procedimento permite detectar e prevenir quadros hipoglicêmicos ou hiperglicêmicos conferindo ao diabético a capacidade de reajustar as doses e tipo de insulina a fim de manter os níveis de glicose sangüíneo o mais próximo possível do normal, evitando ou retardando o aparecimento de complicações agudas e crônicas.

Com o estudo constatamos que a monitorização entre estas crianças e adolescentes diabéticos, está aquém das indicações e recomendações da literatura, quanto a freqüência na monitorização dos níveis de glicose no sangue e urina.

É crucial a implantação e manutenção da educação continuada aos portadores de diabetes, seja para informar ou para corrigir possíveis falhas quanto ao tipo ou técnica para realização do teste de controle, freqüência, avaliação dos valores glicêmicos aferidos, incluindo técnica e preparo de aplicação de insulina. Quanto a estes aspectos, ZANETTI et al.(2001) revelam que compete ao enfermeiro assumir seu papel de educador nas situações em que estão inseridos junto aos pacientes diabéticos de forma mais efetiva.

Neste contexto, a atuação do enfermeiro na equipe multidisciplinar é decisiva para a colaboração e participação do diabético e familiares quanto aos reajustes dos esquemas de tratamento. Sendo que a assistência individualizada garante maior estabilidade emocional a estes indivíduos, auxiliando-os a superar as suas dificuldades sejam no âmbito social, emocional ou econômico.

Dessa maneira, é possível contribuir para uma expectativa de vida maior e, acima de tudo, de melhor qualidade de vida aos portadores de diabetes.

ABSTRACT: The monitorization of the glicemic control is a fundamental part of the treatment of Diabetes Mellitus, specially in type 1 diabetics, as the metabolic control decreases or even delays chronic complications. It was elaborated a describe research with quantity approach which the purpose was appraise the practice of the glicemic monitorization between children and adolescents whose they have been assisted in a interdisciplinary program of the " Ambulatório Hospital das Clínicas da Universidade Estadual de Londrina." An instrument was used to verify the frequency of the accomplishment of the monitorization and the difficulties found for its effectivation. The sample was constituted of 63 diabetics, 47 (74,6\%) adolescents and 16 
$(25,4 \%)$ children. It was concluded that in these population, the monitorization is very distant from the ideal. This information mobilized all persones from the team multiprofessional in the continuity of the work of pointing out the importance of the glicemic control, assisting the diabetics in the search of alternatives for the overcoming of the difficulties from emotional, economical and social order, that make difficult the accomplishment the procedure.

KEY WORDS: Diabetes Mellitus; Diabetic children; Diabetic adolescents; Glicemic monitorization; Monitoring ambulatory.

\section{REFERÊNCIAS}

1. BRASIL. Ministério da Saúde e Secretária de Assistência a Saúde. Departamento de Assistência e Promoção à Saúde. Coordenação de Doenças Crônicas-Degenerativas. Manual de Diabetes. 2. ed. Brasília: Ministério da Saúde, 1993.

2. BRUNNER, L. S.; SUDDARTH. Tratado de enfermagem médico cirúrgico. 8.ed. Rio de Janeiro: Guanabara Koogan, v. 3, 2000. p. 874-884.
3. DUNNE, N.; ZANOWIAK, P.; MELLEN, K. Monitorização da glicose no sangue - o que os pacientes precisam saber. Terapêutica em Diabetes, jul./ago./set., 1995. p.1-3.

4. GOLDSTEIN, D.E et al. Tests of glycemia in diabetes. Diabetes Care, Alexandria, v. 18, n. 6, 1995. p. 896-909.

5. OLIVEIRA, M.L. Insulinoterapia. In: ALMEIDA, H.G.G.L. Diabetes Mellitus: uma abordagem simplificada para profissionais de saúde. São Paulo: Atheneu, 1997, p. 38.

6. PASTORE, K. O inimigo oculto. Veja. São Paulo, 22 jul 1998. p.77.

7. SOUZA, C.R.; ZANETTI, M.L. Administração de insulina: uma abordagem fundamental na educação em diabetes. Revista da Escola de Enfermagem. USP, São Paulo, v. 34, n. 3, set. 2000. p.264-270.

8. ZANETTI, M.L.; MENDES, I.A.C.; RIBEIRO, K.P. O desafio para o controle domiciliar em crianças e adolescentes diabéticas tipo 1. Revista Latinoamericana de Enfermagem, Ribeirão Preto, v. 9, n. 4, jul.2001. p. 32-36.

Endereço das autoras: Rua Odésio Simino, 180 86050-210 - Londrina - PR E-mail: eduenf@uel.br 